\title{
New type of formulations and new application techniques; consequences for the authorisation of pesticides
}

\author{
W. H. M. Ad Meijs
}

Published online: 5 September 2007

(C) Springer Science+Business Media, LLC 2007

\begin{abstract}
The assessment of pesticides is based on the intrinsic properties of the formulation, the ingredients of the formulation and the way the pesticide is applied. The parameters used in the assessment of the efficacy and environmental risks (plants, humans and animals) may be changed by the properties of new pesticides and new application techniques. The type of information needed for new pesticides and application techniques to comply with regulations set up by authorisation bodies will be discussed.
\end{abstract}

\section{Introduction}

Many efforts are taken by interested parties to reduce the risks of pesticides. As well as replacement of old 'unfriendly' pesticide active substances by new 'more friendly' active substances, other developments are found as solutions for risk reduction. These developments can generally be divided into three groups:

- New type of formulations such as aqueous preparations, slow release formulations, granules, special baits, purified biological active isomers.

- New application techniques such as seed coating, cleaning in place, spot techniques, row application, electro-static spray treatment.

- New packaging and dosing techniques such as water soluble packages, use in closed circulation systems, specific dose units.

W. H. M. Ad Meijs ( $₫)$

Board for the Authorisation of Pesticides, P.O. Box 217,

6700 AE Wageningen, The Netherlands

e-mail: a.w.h.m.meijs@ctb.agro.nl
Each type of development influences the hazard and risk assessment of the pesticide in a certain way.

Most of the new developments were started with a view to reduction of the risks for the environment or the user of the pesticides and in this they have been successful.

The regulatory bodies dealing with authorisation of pesticides also have to look at the consequences of new developments from every aspect.

\section{Hazard and risk assessment of pesticides}

The decision on authorisation is based on the hazard and risk assessment from several aspects:

- Efficacy

- Phytotoxicity if used as plant protection product

- Risk for consumer

- Risk for worker, operator and bystander with pesticide or with products applied with the pesticide

- Risk to soil, water and air

- Risk for non-target organisms in the environment

For the authorisation of pesticides hazard assessments of the pesticide and the active substances are made as well as risk assessments of the pesticide for efficacy and each side-effect.

For hazard assessment information is needed on:

- Physical-chemical properties of pesticide and active substance

- Efficacy

- Phytotoxicity

- Toxicity

- Residues

- Fate and behaviour

- Ecotoxicity 
Most of the information is provided as basic studies of the properties of the formulation and the active substances, performed according to established guidelines. The results of the studies lead to the intrinsic properties of the pesticide and active substances. The intrinsic properties, expressed as endpoints for each relevant issue, have to be combined with the exposure to the pesticide as applied for the envisaged use. To calculate these risks further information is needed as follows:

For risk assessment information is needed on:

- Exposure of workers, operators and bystanders

- Exposure of consumers

- Exposure of compartments soil, water and air

- Exposure of non-target organisms in the environment

Most of the information on exposure is derived theoretically using models. The assumptions used in the models are validated by measurements under field circumstances.

The final decision on authorisation is made by comparison of the endpoints (the intrinsic properties) with the exposure.

\section{Influence of the new developments on the authorisation procedure}

Any new development can influence the hazard assessment and/or the risk assessment of the pesticide for one or more aspects. The influence can be positive in some aspects (e.g. more bio-effective; lower risk to earthworms), but at the same time can be negative in other aspects (e.g. higher risk for the consumer). The consequences for each aspect have to be incorporated in the final decision on the admissibility of the pesticide. Using a number of examples the possible influences will be discussed.

3.1 Possible influence on physical-chemical properties of pesticide and active substance

\subsubsection{New formulation}

Many or all properties can be changed when the formulation is changed. Since the physical-chemical properties are very important parameters for the different aspects of the assessment, determination of all physical-chemical properties has to be done thoroughly as relevant information for the calculation of the efficacy and the side-effects of the new pesticide.

\subsubsection{New application, packaging and dosing technique}

Since the formulation is unchanged, consequences for the calculation of efficacy and side-effects are not relevant.
However when using water soluble packages, the physicalchemical properties of the spraying solution can change.

\subsection{Possible influence on efficacy and/or phytotoxicity}

\subsubsection{New formulation}

Efficacy can be influenced by emulsifying properties of the formulation e.g. use of different emulsifiers; adaptation to water-based formulations.

Efficacy can be improved e.g. by slow release formulations.

Phytotoxicity can be increased.

\subsubsection{New application, packaging and dosing technique}

Efficacy can be improved e.g. by row application, plant detection systems or reduced e.g. by increased droplet size.

The new formulation or new application can lead to minimum doses to achieve sufficient efficacy. The new dose influences the assessments of other aspects.

\subsection{Possible influence on risk for consumer}

- Toxicity

- Residues

- Exposure to consumers

\subsubsection{New formulation}

The risk for the consumer can be increased due to higher toxicity of the pesticide (biologically active isomers) or increased exposure (slow release formulation). The risk for the consumer can also be lower due to lower toxicity (biologically active isomers) or lower exposure (granules).

\subsubsection{New application, packaging and dosing technique}

The risk of the pesticide can be increased due to greater exposure to the pesticide (row application, electro-static spray treatment) or decreased due to lower exposure (spot techniques).

Example. Using a row application on strawberries (with equipment facing the strawberry plants) as replacement of a full field application.

The total dose changed from 2 to $1 \mathrm{~kg} / \mathrm{ha}$.

The residue level in the strawberries increased from 1 to $1.5 \mathrm{mg} / \mathrm{kg}$. 
3.4 Possible influence on risk for worker, operator and bystander with pesticide or with products applied with the pesticide

- Toxicity

- Exposure of workers, operators, bystanders

\subsubsection{New formulation}

Pesticide risk can be increased due to higher toxicity of the pesticide (biologically active isomers), or increased exposure (slow release formulations, ultra low volume formulations), not only at the time of application, but also afterwards (crop re-entry, further activities in/within treated rooms/materials).

Pesticide risk can be reduced due to lower toxicity of the pesticide (biologically active isomers) or reduced exposure (slow release formulation, water soluble packages, granules), not only at the moment of application, but also afterwards (crop re-entry, further activities in/within treated rooms/materials).

\subsubsection{New application, packaging and dosing technique}

Pesticide risk can be greater due to increased exposure to the pesticide (ultra low volume) or reduced (larger sized droplets, protection screens, circulation systems, spot techniques).

Example. EUROPOEM is in development now as model for the calculation of the risk for worker. In this model parameters used are duration of oral, dermal and inhalation exposure; particle size of dusts; particle size of respiratory fractions; percentage dermal absorption; room concentration.

Many of these parameters can change due to new types of formulation or application techniques.

3.5 Possible influence on risk for the soil, water and air

- Fate and behaviour

- Exposure of compartments soil, water and air

\subsubsection{New formulation}

Pesticide risk can be increased due to higher toxicity (unfavourable endpoints) of the pesticide (biologically active isomers) or higher exposure (slow release formulation, ultra low volume formulations, granules, baits), not only at the moment of application, but also afterwards (degradation time).

Pesticide risk can be reduced due to lower toxicity (favourable endpoints) of the pesticide (biologically active isomers) or lower exposure (slow release formulation, water soluble packages, granules), not only at the moment of application, but also afterwards (degradation time, frequency of use).

\subsubsection{New application, packaging and dosing technique}

Pesticide risk can be greater due to increased exposure to the pesticide (ultra low volume) or reduced (larger size droplets, protection screens, circulation systems).

Example. PEARL is used as one of the FOCUS-models for the calculation of the leaching of a pesticide to groundwater. Parameters such as DT50 and Kom are used in the model to calculate the leaching of $1 \mathrm{~kg} / \mathrm{ha}$ of active substance.

Afterwards the calculated leaching is corrected for the effective amount of active substance reaching the soil, using the following formula:

$C=f \cdot[(100-V-1) / 100] \cdot D$

$C$-Effective amount in the soil $(\mathrm{kg} / \mathrm{ha})$

$F$-Frequency of use

$V$-Volume \%, wasted during spray application

$I$-\% interception by the crop

$D$-Dose (single use, $\mathrm{kg} / \mathrm{ha}$ )

All these parameters i.e. frequency $(f)$, wasted volume $(V)$, interception \% $(I)$ and dose $(D)$ can change due to a new type of formulation or application technique.

3.6 Possible influence on risk for non-target organisms in the environment

- Ecotoxicity

- Exposure of non-target organisms in the environment

\subsubsection{New formulation}

Pesticide risk can be increased due to higher toxicity of the pesticide (biologically active isomers, seed coating formulations) or greater exposure (slow release formulation, ultra low volume formulation, granules), not only at the moment of application, but also afterwards (slower degradation).

Pesticide risk can be reduced due to lower toxicity of the pesticide (biologically active isomers) or reduced exposure (slow release formulation), not only at the moment of 
application, but also afterwards (lower frequency of applications).

\subsubsection{New application, packaging and dosing technique}

Pesticide risk can be increased due to higher exposure to the pesticide (ultra low volume, row application) or reduced (larger droplet size, protection screens, circulation systems).

Example. Using TOXSWA as model for the calculation of the risk for aquatic organisms:

$\mathrm{PEC}=\mathrm{EC} \cdot \%$ drift dose

PEC-Predicted environmental concentration (realistic exposure concentration) in $\mu \mathrm{g} / \mathrm{l}$

EC-Momentary concentration or TWAEC as determined from a standard deposition in $\mu \mathrm{g} / \mathrm{l}$

$\%$ drift-Percentage drift deposition

dose-Application rate in $\mathrm{kg}$ active substance/ha

TWAEC is the time weighed average exposure concentration over 4,21 or 28 days. TWAEC has to be determined from graphs as a function of the overall dissipation half-life time DT50, accounting for transformation and volatilisation.

Taking a slow release formulation: the PEC-value can be influenced through the parameters momentary concentration (EC), dose, TWAEC (due to other dose, other degradation profile, other time interval of application).

\section{Conclusion}

New developments for risk reduction on one assessmentaspect of a pesticide can influence the other aspects of the pesticide. The influence can be positive in one aspect (for example more effective, lower risk for earthworms), but at the same time the influence can be negative for one or more of the other aspects (higher risk for the consumer, birds).

The consequences for each aspect have to be incorporated in the final decision of the total validity of the envisaged change.

The authorisation bodies as well as the companies have to consider this also.

Authorisation bodies and companies have to consider also the consequences of the new types of pesticides and the new techniques for the models used to calculate the risks of the pesticides.

Applicants have to submit new data for all the aspects, which are changed by the new formulation or the new technique. They always have to take into account the consequences of their improvements on one aspect for the assessments of the other aspects. 